\title{
Nursing Experience of Vena Cava Filter Combined with Catheter Thrombolysis in the Treatment of Deep Venous Thrombosis of Lower Limbs
}

\author{
Yuefei Li \\ Army Medical Center of PLA, Chongqing 400042, China \\ Email: 136876899@qq.com
}

\begin{abstract}
Objective - To observe the curative effect of urokinase catheter thrombolysis in treating deep venous thrombosis of lower limbs after implantation of vena cava filter and explore nursing measures and nursing points. Methods - Perioperative nursing of 30 deep venous thrombosis patients with catheter thrombolysis treatment were summarized retrospectively. Results - 30 patients were conducted with catheter pumped urokinase to treat venous thrombus, and the swelling and pain of limbs disappeared. No patient had pulmonary embolism or hemorrhage. Conclusion - When we use urokinase catheter thrombolysis to treat deep venous thrombosis, a comprehensive preparation such as health education, psychological nursing, close observation on patients' condition, proper fixation of catheter, nursing of thrombolytic drugs and prevention of infection can reduce the occurrence of complications and promote the patients rehabilitation.
\end{abstract}

Keywords: deep venous thrombosis of lower limbs, catheter, thrombolysis, nursing

Deep venous thrombosis of lower limbs is a common vascular surgical disease, accounting for about $95 \%$ of peripheral venous occlusive diseases. The clinical manifestations are swelling, pain, superficial varicose veins and systemic reactions of the affected limbs. If the treatment is not timely or improper, edema, varicose veins, skin ulcer, etc. May occur, affecting the daily activities of patients, and serious cases may lead to complications such as pulmonary embolism ${ }^{[1]}$. Vena cava filter implantation plus catheter contact thrombolysis is a new technique, which can not only prevent the thrombus more than $3 \mathrm{~mm}$ from entering the pulmonary artery to cause pulmonary embolism, but also do not affect venous reflux, and has the advantages of less complications, less injury, less bleeding, rapid recovery, short hospital stay and so on ${ }^{[2]}$. In the clinical treatment process of the disease, nursing work plays a great auxiliary role, and even directly affects the clinical curative effect. In October 2012 and October 2015, 30 patients with deep venous thrombosis of lower limbs were treated in our hospital, which were treated with vena cava filter implantation combined with popliteal vein puncture catheter thrombolysis treatment. After the operation, anti-inflammatory, thrombolytic, anticoagulant and other treatments were given, and nursingful nursing was given, which achieved satisfactory results. The report is as follows.

\section{Clinical data}

\subsection{General information}

There were 30 patients, 12 males and 18 females, aged from 20 to 78 years old, with an average age of 55 years. The course of disease was 3 10 days, including 20 cases of deep venous thrombosis in left lower limb and 10 cases of deep venous thrombosis in right lower limb. The clinical manifestations were swelling, pain, dark purple and red skin, and skin temperature elevation in some patients.all patients were diagnosed by color Doppler ultrasound or lower limb phlebography before operation.patients with femoral, popliteal and iliac vein trunk full limb thrombosis were included in this group.

\subsection{Treatment methods}

Under digital subtraction angiography (DSA), routine disinfection, local anesthesia with 1\% lidocaine, femoral vein puncture and insertion of $6 \mathrm{f}$ sheath through the groin of the healthy side by Seldinger technique, angiography to evaluate the renal vein opening and inferior vena cava whether there is deformity, stenosis, thrombosis, blockage, inferior vena cava filter insertion. After the filter was placed successfully, the vascular sheath was pulled out, the puncture site was pressed for 10 15 minutes, and sterile gauze was covered. After the filter is arranged, the patient is turned over and subjected to superficial vein puncture under the guidance of ultrasound, a thrombolysis catheter is placed in the thrombus part, the silk thread is fixed on the surface of the skin, the three-way switch is placed in a closed state after the catheter is sealed with 
heparin normal saline, and then the three-way switch is sent to the ward. Urokinase was pumped continuously at a constant speed, the total dose was 60-80 Wu for 24 hours, and the total dose was 300-400 WU, with an average of 350 Wu for 3 5 days. Low molecular weight heparin was injected subcutaneously twice a day, and warfarin (adjusted INR 1.5-2) was taken 3 5 days before discharge, and rivaroxaban was taken on the eve of discharge. All the 24 patients with temporary filter placement returned to hospital 2 weeks after discharge to remove the temporary filter. Patients were required to take anticoagulants orally for more than half a year after discharge.

\subsection{Results}

Vena cava filters were placed $1 \sim 2 \mathrm{~cm}$ below the renal vein opening in all 30 patients, including Johnson permanent filters in 6 cases and retrievable filters OptEaseTM filter in 24 cases. The position of the filter was good without displacement, and the average thrombolysis time was 3 5 days. Venography of the affected limbs showed smooth vein wall and uniform contrast agent density in 7 cases, and local filling defect was found in 23 cases, of which 20 cases had open lateral branches. Further interventional therapy was performed for the findings of Cockett's syndrome. At the time of discharge, the swelling of the limbs subsided significantly and the pain disappeared. Of the 24 temporary filters, 22 were removed successfully two weeks after discharge, 1 gave up removal because of vena cava thrombosis, and 1 gave up removal because of overdue. In this group, 24 cases were cured, 6 cases were effective, no pulmonary embolism, bleeding and other complications occurred, postoperative follow-up of 3 to 6 months no new thromboembolism occurred, swelling discomfort basically disappeared.

\section{Nursing experience}

\subsection{Preoperative nursing}

Psychological nursing: Due to the acute onset of the disease, typical clinical symptoms, high treatment costs, and slow elimination of the edema of the affected limbs, the patient is worried about the metal stent inserted into the vena cava filter, and the psychological pressure is high. The medical staff should be patient with the patient and family. The nursing staff should introduce the purpose, methods, characteristics, precautions, etc. of the operation to patients and their families in detail, focusing on successful cases of surgery, improve patients and their families' awareness of thrombolytic therapy, and reduce the psychological burden on patients and their families. The trust of patients and their family members eliminates their anxiety and anxiety, actively cooperates with the operation, and improves the treatment effect.

General nursing: Assist the patient to raise the affected limb by 20 to 30 degrees to facilitate venous return, reduce venous pressure, and reduce swelling. Pay attention to keeping the limbs warm, which can speed up blood circulation and relieve pain. In the acute phase, patients should stay in bed absolutely, massage the affected limbs are forbidden, to prevent emboli from falling off and cause pulmonary embolism, and ask the patients whether they have pain in the chest area, chest tightness and other discomforts. Instruct patients to avoid forced defecation to increase abdominal pressure, which affects the venous blood return of the lower extremities. Avoid tobacco and alcohol, reduce nicotine contraction of peripheral blood vessels, and promote blood circulation. Before surgery, check heart, lung, liver, and kidney functions, blood routines, and clotting time. Skin preparation: from below the navel to $1 / 3$ of the thigh, including the perineum and affected limbs, clean the skin, pay special attention to whether there is skin disease, damage or infection at the puncture site, and train the patient to urinate and urinate on the bed. Before the operation, accurately measure the skin temperature and the circumference of the lower limbs at the same position, and observe the color of the lower limbs for comparison with the postoperative.

\subsection{Postoperative nursing}

General nursing: clarify the puncture site, intraoperative conditions, etc. After the operation, the patient was placed in a bed unit not far from the nurse's station, rested in bed, and the affected limb was raised by 20 to 30 degrees. Closely observe the changes in vital signs and oxygen saturation. If the patient is found to have suspicious pulmonary embolism or bleeding symptoms in the digestive tract, urinary system, etc., report to the doctor for treatment in time. Instruct patients to eat more low-fat, high-protein, high-fiber fresh vegetables and fruits, encourage drinking more water, and eat less spicy and irritating foods. Non-smoking alcohol to prevent the nicotine in the smoke from stimulating vasospasm. Advise the patient to drink more water to reduce the viscosity of blood pressure and promote the excretion of the contrast agent. At the same time, observe whether the urine output is roughly the same as the drinking water to prevent renal failure.

Limb nursing: Do not bend or curl up on the side of the puncture limb, and leave it in a straightened position for 12 hours. Observe the patient's limb skin color, temperature, dorsal artery pulsation, limb swelling subsided, and the 
patient's feeling to understand the effect of thrombolysis. If the skin becomes pale, blue, chills, dorsal artery pulsation disappears, limb swelling, and pain worsens, immediately notify the doctor for treatment. Instruct the patient to take the supine or semi-sitting position, the affected limb is raised to the heart level by 20 to $30 \mathrm{~cm}$, and can be used for toe foot dorsiflexion and ankle joint activities to facilitate venous return and reduce swelling. After the limb brake is eliminated, the patient should move as soon as possible and encourage the patient to move independently on the bed. After the catheter is removed, the patient can get out of bed and move around after 24 hours of compression bandaging to avoid new thrombosis.

Catheter nursing: The thrombolytic catheter usually takes 3 5 days. The catheter slips out easily during activities. Evaluate the safety of the catheter and formulate nursing measures. Instruct patients that the range of motion on the bed should not be too large, fix the catheter properly, fix it with a $8^{*} 10 \mathrm{~cm}$ transparent applicator at the puncture site, avoid joints and depressions, and wrap it with elastic bandages. Because the outer part of the catheter is longer, prevent the catheter from being twisted, compressed, and buckled when it is flexed and fixed. If the catheter is found to move out, notify the doctor immediately. Clean and change the dressing every $2 \mathrm{~d}$ to keep the skin around the catheter clean and dry to prevent ascending infection and complicated phlebitis. Distinguish between sheath and thrombolytic catheter to prevent wrong medication route. The outer end of the catheter is sealed with a heparin cap, the infusion pump, extension tube, and thrombolytic catheter are correctly connected, and the joints are checked for tightness. Replace with new extension tubes and infusion tubes every 24 hours.

Thrombolysis and anticoagulation nursing: Urokinase thrombolysis is the most critical step in the treatment, which is directly related to the effect of thrombolytic therapy ${ }^{[3]}$. Urokinase should be stored in the refrigerator, and should not be diluted with acidic liquids, so as not to reduce the efficacy of the drug, and it is easy to inactivate after dissolution, so it is ready for immediate use. The thrombolytic catheter is routinely indwelled for 3 to 5 days after the operation. In accordance with the doctor's instructions, $50 \mathrm{ml}$ of $0.9 \%$ sodium chloride and $20 \mathrm{U}$ of urokinase are injected with a 99 $\mathrm{ml} / \mathrm{h}$ micro pump. The thrombolytic catheter, extension tube and infusion pump are correctly connected. Whether the thrombolytic catheter is pumped into urokinase smoothly, whether the speed is appropriate, whether the dose meets the actual requirements, always observe whether the infusion pump dripping speed is appropriate, and if an alarm occurs, immediately find the cause and eliminate the fault. During the process of thrombolysis, monitor the prothrombin time, assist in the re-examination of deep venography, to understand the effect of thrombolysis, and adjust the position of the catheter in time.

Complication nursing: (1) Pulmonary embolism. Pulmonary embolism is one of the most serious complications of deep venous thrombosis of the lower extremities. Thrombolytic therapy can quickly and effectively dissolve the thrombus, but the incompletely dissolved thrombus is detached from the blood vessel wall. Even after the inferior vena cava filter is inserted, $2 \%$ to $6 \%$ of patients can develop pulmonary embolism. Instruct patients to stay in bed after surgery to prevent coughing, constipation, etc., and to prevent emboli from falling off. Give ECG monitoring, closely observe whether the patient has pulmonary $\mathrm{P}$ wave, T wave inversion, ST segment changes and changes in respiration, oxygen saturation, and vital signs, such as sudden severe pain, shortness of breath, cyanosis, cough, hemoptysis Wait for the symptoms of pulmonary embolism, assist the patient to take the supine position, avoid deep breathing, coughing, and violent turning, and at the same time give high-concentration oxygen inhalation of 6 to $8 \mathrm{~L} / \mathrm{min}$, report to the doctor, and actively cooperate with the rescue ${ }^{[4]}$. None of the patients in this group had pulmonary embolism. (2) Bleeding. In order to treat thrombosis and prevent thrombosis from forming again, continue to use anticoagulant thrombolytic drugs after the operation to make the body in a hypocoagulable state, which may cause bleeding. The most common is blood oozing at the puncture site, and various nursing operations should be gentle to avoid injury. Venous puncture strives to be successful at one time. After removing the needle, press for 10 minutes, observe the dressing at the puncture site, and use compression to stop bleeding if necessary. Pinch the skin when subcutaneously injecting low-molecular-weight heparin, pay attention to avoiding the subcutaneous blood vessels, and increase the local pressing time after injection. Instruct patients to brush their teeth with a soft toothbrush, and rinse their mouth with cold water if they find bleeding gums. Closely observe the patient's pupils, consciousness and other intracranial hemorrhage, pay attention to observing the bleeding of the digestive tract and urinary tract such as the color and character of the urine and stool, and recheck the prothrombin time daily to maintain it in the range of 1.5 to 2.5 times the normal level, Report to the doctor promptly if any abnormality is found. 6 people in this group had bleeding at the puncture site, which was relieved after adjustment of thrombolytic drugs and local treatment. (3) Catheter slip thrombolytic catheter is generally indwelled for 5 to 7 days. If the thrombolytic catheter is not firmly fixed after the operation, the patient is prone to catheter slip due to large mobility. Therefore, the catheter should be properly fixed and the length of the catheter left outside the body should be recorded. If the catheter is moved outside, it should 
not be sent into the blood vessel to cause infection. If necessary, use a strong elastic bandage to paste, make the bedside handover of each shift, and check whether the joints of the catheters are tight. There was no catheter slippage in this group.

Extubation nursing: remove it within 5-7 days after surgery, routinely disinfect with alcohol and then pull out the thrombolytic catheter, press the puncture site for 15-20 minutes, apply a strong elastic bandage to pressurize it, and ask the patient to immobilize the affected limb 8 hours, stay in bed absolutely for 24 hours, and observe whether there is any bleeding at the puncture site, the pulsation of the dorsal foot artery of the limbs, skin color, humidity and capillary filling time, and ask for pain, sensory disturbance and swelling, etc., if unwell, promptly Report to the doctor.

\subsection{Discharge guidance}

Excessive activities should not be performed 1 month after discharge. Pay attention to step by step to prevent lower extremity edema. After 3 months, avoid weight-bearing activities and insist on functional exercises. Avoid standing for a long time and walking for a long time, or tilt your "erlang's legs" to prevent blood vessels from being compressed. Elevate the affected limb to reduce venous swelling, and avoid collision with the affected limb that will affect wound healing. No smoking, alcohol, to prevent nicotine from stimulating vasospasm. Pay attention to keeping the affected limb warm to prevent excessive cold from stimulating vasoconstriction and affecting blood supply. Instruct patients to take oral anticoagulants for more than half a year, regularly review the coagulation function, measure the international standardized ratio every week in the first month and maintain it between 2 and 3, and start a monthly review in the second month. Nonsmoking alcohol, continue a low-salt, low-fat diet. Eat more fruits and cellulose-rich foods, drink plenty of water, and maintain smooth stools. Regularly review the X-ray to observe the position of the filter, whether there is displacement, etc., and tell the patient the methods of self-assessment and monitoring. If there are bleeding spots on the skin and mucous membranes or bleeding from the gums, stop the medication immediately and return to the hospital for diagnosis and treatment.

\section{Discussion}

Acute deep venous thrombosis (DVT) is a common clinical disease. If it is not treated in time, it will affect the ability to live and work, and even cause disability. Pulmonary embolism is the most serious complication of deep venous thrombosis of the lower extremities. Improper treatment and nursing can easily cause embolism to fall off and cause acute embolism of the pulmonary artery, which can lead to death of the patient. The main treatment for deep venous thrombosis is anticoagulation. The drugs used include heparin and warfarin. For some patients, especially for thrombosis of the whole limbs, anticoagulation alone is difficult to achieve the desired therapeutic effect. It is necessary to consider the dissolution after the insertion of the vena cava filter. Adjuvant treatment with suppository or surgical incision to remove the suppository ${ }^{[5]}$. DVT catheter-directed thrombolysis (CDT) is the use of Seldinger technology to insert a thrombolytic catheter into the thrombotic vein under X-ray or ultrasound guidance, usually through the popliteal vein of the affected limb. The micro-injection pump is used to continuously inject the fibrinolytic drug, so that the drug is in full contact with the thrombus, and the purpose of dissolving the thrombus is achieved. In 1999, 63 research centers in the United States used thrombolytic catheters to inject urokinase in the treatment of 287 cases of iliofemoral and femoral popliteal DVT. It is believed that this method is safe and effective in the treatment of lower extremity DVT, and is considered to be an acute limb rescue method ${ }^{[6]}$. In the process of direct thrombolysis, it prevents the thrombus or the decomposed small thrombus from falling off, causing the embolism of important organs. In the case of this study group, the inferior vena cava filter was inserted first (temporary vena cava filter is preferred and taken out after 21 days), followed by direct urokinase pumping thrombolytic therapy through the insertion of the catheter, which can better prevent and treat the symptoms of deep venous thrombosis of the lower extremities. And complications. No pulmonary embolism occurred in this group of cases after the filter was placed. On this basis, meticulous psychological and physical nursing, complete health education, enabling patients to fully grasp the knowledge of self-nursing, can help patients build confidence in overcoming the disease, help reduce bleeding, re-embolization and disability, and help reduce the pressure of the society and the family.

\section{References}

[1] Vascular Surgery Group of Surgery Branch of Chinese Medical Association. Guidelines for the diagnosis and treatment of deep venous thrombosis (2nd edition)[J]. Chinese Journal of Surgery, 2012, 50 (7): 611-612.

[2] Zhang Qingyun, Gao Jianguo, Chen Yong, et al. Application of inferior vena cava filter implantation and catheter thrombolysis in the treatment of deep venous thrombosis of the lower extremities[J]. Journal of the Third Military 
Medical University, 2010, 32(17): 1887.

[3] Fang Liyan, Lang Dehai, Hu Songjie. Nursing nursing of deep venous thrombosis of lower limbs treated with thrombolysis via popliteal vein catheter[J]. Nursing and Rehabilitation, 2010, 9(9): 782.

[4] Wang Xianmei. Observation and nursing of interventional treatment of deep venous thrombosis of lower extremities [J]. Knowledge of Cardiovascular Disease Prevention and Treatment, 2014, 9(6): 82-85.

[5] Vedantham S. Interventional approaches to deep venous thrombosis[J]. Am J Hematol, 2012, 87 (Suppl) 1: S113.

[6] Herrera S, Comerota AJ. Embolization during treatment of deep venous thrombosis:incidence, importance, and prevention[J]. Tech Vasc Interv Radiol, 2011, 14(2): 58-64. 